PROCEEDINGS OF THE

AMERICAN MATHEMATICAL SOCIETY

Volume 125, Number 9, September 1997, Pages 2771-2775

S 0002-9939(97)04172-5

\title{
THE SPACE OF SUBCONTINUA OF A 2-DIMENSIONAL CONTINUUM IS INFINITE DIMENSIONAL
}

\author{
MICHAEL LEVIN AND YAKI STERNFELD
}

(Communicated by James West)

\begin{abstract}
Let $X$ be a metric continuum and let $\mathcal{C}(X)$ denote the space of subcontinua of $X$ with the Hausdorff metric. We settle a longstanding problem showing that if $\operatorname{dim} X=2$ then $\operatorname{dim} \mathcal{C}(X)=\infty$. The special structure and properties of hereditarily indecomposable continua are applied in the proof.
\end{abstract}

\section{INTRODUCTION AND PRELIMINARIES}

Let $X$ be a compact metrizable space. $2^{X}$ denotes the space of closed subsets of $X$ endowed with the Hausdorff metric. $2^{X}$ is compact. $\mathcal{C}(X)$ is the closed subset of $2^{X}$ that consists of the subcontinua of $X$. In this note we prove that if $\operatorname{dim} X=2$ then $\operatorname{dim} \mathcal{C}(X)=\infty$. This settles a longstanding open problem. (See [6] in particular p.217 and 226-227) It was known ([4], Nadler, Rogers, see [6]) that if $\operatorname{dim} X \geq 2$ and $X$ is a hereditarily indecomposable continuum then $\operatorname{dim} \mathcal{C}(X)=\infty$. As by [1] every continuum of $\operatorname{dim} \geq 3$ contains some hereditarily indecomposable continuum of $\operatorname{dim} \geq 2, \operatorname{dim} X \geq 3$ implies $\operatorname{dim} \mathcal{C}(X)=\infty$, and our result improves this. (See also [3] for another result which implies $\operatorname{dim} \mathcal{C}(X)=\infty$ for 2-dimensional $X$ which satisfies some additional conditions.) Our proof applies some of the ideas of the earlier proofs as well as the results of [1]. In particular our Lemma 1.3 was inspired by Theorem 7.8 of [4]. We wish to thank H. Kato for introducing this problem to us. The paper is self-contained modulo the results of [1]. In this section we present some preliminary results which may be of some independent interest, and in Section 2 we prove the main result and a stronger version. All spaces in this note are assumed to be separable metric.

Theorem 1.1. Let $X$ be an $n$-dimensional compact metric space, $n<\infty$. There exist an $n$-dimensional hereditarily indecomposable continuum $Y$ and a light map $f$ of $Y$ into $X$.

Proof. $\operatorname{dim} X \times I=n+1, I=[0,1]$. By [1] there exists an $n$-dimensional hereditarily indecomposable continuum $Y \subset X \times I$. Let $P: X \times I \longrightarrow X$ be the projection, and set $f=\left.P\right|_{Y} . f$ is light since a component of a fiber of $f$ is a subcontinuum of both $Y$ and $I$ and hence must be a singleton.

Received by the editors February 4, 1995 and, in revised form, March 31, 1996.

1991 Mathematics Subject Classification. Primary 54B20, 54F15, 54F45.

Key words and phrases. Hyperspaces, hereditarily indecomposable continua, 2-dimensional continua. 
Remark. Note that it follows that dim. type $Y \leq \operatorname{dim}$. type $X$ in the sense of [2] and also that $\operatorname{dim} f(Y)=n$.

Definition 1.2 (see [6]). Let $X$ be a continuum. A map $W: \mathcal{C}(X) \longrightarrow \mathbb{R}^{+}$is called a Whitney map if $W$ vanishes on the set of singletons in $\mathcal{C}(X)$ and if $A \neq B$ in $\mathcal{C}(X)$ and $A \subset B$ implies $W(A)<W(B)$.

Whitney maps always exist: if $\left\{f_{n}\right\}_{n=1}^{\infty}$ is a dense sequence of functions in $C(X,[0,1])$ and $W_{n}(A)=\operatorname{diam} f_{n}(A), W_{n}: \mathcal{C}(X) \longrightarrow[0,1]$ then $W=\sum_{n=1}^{\infty} W_{n} / 2^{n}$ is a Whitney map.

Let $d_{n}(Z)$ denote the $n$-dimensional degree as defined in [5] p. 105. Recall that $d_{n+1}(Z)<\epsilon$ if and only if $Z$ admits a finite open cover of order $\leq n$ and mesh $<\epsilon$. Hence for $Z$ compact $d_{n+1}(Z)=0$ is equivalent to $\operatorname{dim} Z \leq n$, and $d_{1}(Z)<\epsilon$ if and only if the diameter of every component of $Z$ is less than $\epsilon$.

Lemma 1.3. Let $\mathcal{K} \subset \mathcal{C}(Y)$ be a decomposition of a compact metric space $Y$ such that $\inf \{\operatorname{diam} K: K \in \mathcal{K}\}>0$. Let $f$ be a light map of $Y$ into some continuum $X$. Define $g: Y \longrightarrow \mathcal{C}(X)$ by $g(y)=f(K)$ where $y \in Y$ and $K$ is the element of $\mathcal{K}$ which contains $y$. If $g$ is continuous and if $g(Y)$ is a finite dimensional subset of $\mathcal{C}(X)$, then for every $\epsilon>0$ there exists a closed subset $Z$ of $Y$ which intersects every element of $\mathcal{K}$ such that $d_{1}(Z)<\epsilon$.

We prove Lemma 1.3 after making the following remarks (which are well known and are included for completeness).

Remarks. 1. A decomposition $\mathcal{K}$ is closed in $\mathcal{C}(Y)$ if and only if $\mathcal{K}$ is upper semicontinuous and the corresponding quotient map $q: Y \longrightarrow \mathcal{K}$ is open and monotone (see [5] p. 68).

2. If $\mathcal{K}$ is closed in $\mathcal{C}(Y)$ then it is compact and hence $\inf \{\operatorname{diam} K: K \in \mathcal{K}\}>0$ if $\mathcal{K}$ does not contain singletons.

3. If $\mathcal{K}$ is closed in $\mathcal{C}(Y)$ then $g$ is automatically continuous. Indeed, let a sequence $y_{1}, y_{2}, \ldots \in Y$ converge to $y_{0} \in Y$ and let $y_{i} \in K_{i} \in \mathcal{K}, i \geq 0$. Since $\mathcal{K}$ is compact one can find a subsequence $K_{i_{j}}, i_{j}>0$, converging in $2^{Y}$ to some $K \in \mathcal{K}$. Then $y_{0} \in K$ and therefore $K=K_{0}$. Clearly $f\left(K_{i_{j}}\right) \longrightarrow f(K)$ in $2^{X}$. So $g\left(y_{i_{j}}\right)=f\left(K_{i_{j}}\right) \longrightarrow f(K)=f\left(K_{0}\right)=g\left(y_{0}\right)$ and $g$ is continuous.

Proof of Lemma 1.3. As $\mathcal{H}=g(Y)$ is finite dimensional, there exists an integer $N$ (which depends on $\operatorname{dim} \mathcal{H}$ ) such that every open cover of $\mathcal{H}$ has an open refinement $\mathcal{V}_{1}, \mathcal{V}_{2}, \ldots, \mathcal{V}_{m}$ so that each $\mathrm{cl} \mathcal{V}_{i}$ intersects at most $N$ of the other $\mathrm{cl} \mathcal{V}_{j}$. (This is Lemma 7.7 of [4]. One way to obtain $N$ is by embedding $\mathcal{H}$ in a Euclidean space.)

Let $\epsilon>0$ and let $\delta>0$ be sufficiently small such that the following hold:

(i) For $T \subset X$ with $\operatorname{diam} T \leq \delta, d_{1}\left(f^{-1}(T)\right)<\epsilon$ (such a $\delta$ exists since $f$ is light).

(ii) If $B_{1}, B_{2}, \ldots, B_{N}$ are $N$ subsets of $X$ with $\operatorname{diam} B_{i}<3 \delta$, and $A \in \mathcal{H}$ then $A \backslash \bigcup_{i=1}^{N} B_{i} \neq \emptyset$. (Note that as $\inf \{\operatorname{diam} K: K \in \mathcal{K}\}>0$ and $f$ is light, $\lambda=$ $\inf \{\operatorname{diam} H: H \in \mathcal{H}\}=\inf \{\operatorname{diam} f(K): K \in \mathcal{K}\}>0$ and we take $\delta<\lambda / 3 N$ which does the job since a continuum $A$ of diameter $\geq \lambda$ cannot be covered by $N$ sets of diameter $<\lambda / N$.)

Now let $\left\{\mathcal{V}_{1}, \mathcal{V}_{2}, \ldots, \mathcal{V}_{m}\right\}$ be a closed cover of $\mathcal{H}$ with mesh $<\delta$ (mesh with respect to the Hausdorff metric in $\mathcal{C}(X))$ such that each $\mathrm{cl}_{i}$ intersects at most $N$ of the other $\mathrm{cl} \mathcal{V}_{j}$. Note that

(iii) For each $1 \leq i \leq m$, for each $A \in \mathcal{V}_{i}$ and every $x \in A, B(x, \delta)(=$ closed $\delta$-ball in $X$ with center at $x$ ) intersects every $B \in \mathcal{V}_{i}$ (since otherwise the Hausdorff distance between $A$ and $B$ would be more than $\delta$ ). 
Now we construct the required closed subset $Z$ of $Y$. We shall construct inductively closed mutually distinct subsets $W_{i}, 1 \leq i \leq m$, of $Y$ with $d_{1}\left(W_{i}\right)<\epsilon$ such that $W_{i}$ intersects every $K \in \mathcal{K}$ for which $f(K) \in \mathcal{V}_{i}$. Then we take $Z=\bigcup_{i=1}^{m} W_{i}$. $Z$ intersects each $K \in \mathcal{K}$ since the $\mathcal{V}_{i}$ cover $\mathcal{H}$, and $d_{1}(Z)<\epsilon$ since $d_{1}\left(W_{i}\right)<\epsilon$ and $W_{i}$ are mutually distinct closed sets.

To construct $W_{1}$ pick some $A_{1} \in \mathcal{V}_{1}$ and $x_{1} \in A_{1}$. Set $W_{1}=f^{-1}\left(B\left(x_{1}, \delta\right)\right) \cap$ $g^{-1}\left(\mathcal{V}_{1}\right)$. Assume that mutually disjoint sets $W_{1}, W_{2}, \ldots, W_{j-1}$ were constructed as $W_{i}=f^{-1}\left(B\left(x_{i}, \delta\right)\right) \cap g^{-1}\left(\mathcal{V}_{i}\right)$ where $x_{i} \in A_{i} \in \mathcal{V}_{i}, 1 \leq i \leq j-1$. Let $A_{j} \in \mathcal{V}_{j}$. At most $N$ of $\mathcal{V}_{i}, 1 \leq i \leq j-1$, intersect $\mathcal{V}_{j}$. Assume these are $\mathcal{V}_{i_{1}}, \mathcal{V}_{i_{2}}, \ldots, \mathcal{V}_{i_{N}}$. By (ii) let $x_{j} \in A_{j} \backslash \bigcup_{l=1}^{N} B\left(x_{i_{l}}, 3 \delta\right)$. Then $B\left(x_{j}, \delta\right) \cap B\left(x_{i_{l}}, \delta\right)=\emptyset$ for all $1 \leq l \leq N$. Set $W_{j}=f^{-1}\left(B\left(x_{j}, \delta\right)\right) \cap g^{-1}\left(\mathcal{V}_{j}\right)$. Then $W_{j} \cap W_{i}=\emptyset$ for all $1 \leq i \leq j-1$. Indeed, if $i=i_{l}$ for some $1 \leq l \leq m$ this follows from $B\left(x_{j}, \delta\right) \cap B\left(x_{i_{l}}, \delta\right)=\emptyset$ while if $i$ does not belong to $\left\{i_{1}, i_{2}, \ldots, i_{N}\right\}$ it holds since $\mathcal{V}_{i} \cap \mathcal{V}_{j}=\emptyset$. By (i) $d_{1}\left(W_{j}\right)<\epsilon$ and by (iii) $W_{j}$ intersects every $K \in \mathcal{K}$ such that $f(K) \in \mathcal{V}_{j}$. This concludes the proof of the lemma.

The following simple observation will be applied in our proof.

Observation. Let $Y$ be an $n$-dimensional compact space. There exist closed distinct subsets $F_{1}$ and $F_{2}$ of $Y$ and $r>0$ such that every closed subset $L$ of $Y$ which separates $F_{1}$ from $F_{2}$ must satisfy $d_{n-1}(L) \geq r$.

Proof. Let $H_{1}$ and $H_{2}$ be closed disjoint subsets of $Y$ which cannot be separated in $Y$ by a closed subset of dimension $\leq n-2$. If each separator $L \subset Y$ between $H_{1}$ and $H_{2}$ satisfies $d_{n-1}(L) \geq 1$ then we are done. Otherwise let $L_{1} \subset Y$ be a closed separator with $d_{n-1}\left(L_{1}\right)<1$. Let $U_{1}$ be an open neighbourhood of $L_{1}$ in $Y$ with $d_{n-1}\left(U_{1}\right)<1$ and $U_{1} \cap\left(H_{1} \cup H_{2}\right)=\emptyset$. Then $U_{1}$ separates $H_{1}$ from $H_{2}$ and hence $Y \backslash U_{1}=H_{1}^{2} \cup H_{2}^{2}, H_{1} \subset H_{1}^{2}, H_{2} \subset H_{2}^{2}$ and $H_{i}^{2}$ closed in $Y$. If each separator $L_{2}$ between $H_{1}^{2}$ and $H_{2}^{2}$ satisfies $d_{n-1}\left(L_{2}\right) \geq 1 / 2$ then we are done. Otherwise we find a closed separator $L_{2}$ contained in an open separator $U_{2}$ with $\operatorname{cl} U_{2} \subset U_{1}$ and $d_{n-1}\left(U_{2}\right)<1 / 2$, and continue by an obvious induction. We obtain a decreasing sequence $U_{k}$ with $\operatorname{cl} U_{k+1} \subset U_{k}$ and $d_{n-1}\left(U_{k}\right)<1 / k$. Then $L=\cap U_{k}$ is a separator between $H_{1}$ and $H_{2}$, and $d_{n-1}(L)=0$ i.e. $\operatorname{dim} L \leq n-2$ which is a contradiction.

\section{2. $\operatorname{dim} \mathcal{C}(X)$}

Theorem 2.1. Let $X$ be a 2 -dimensional continuum. Then $\operatorname{dim} \mathcal{C}(X)=\infty$.

Proof. By Theorem 1.1 there exist a 2-dimensional hereditarily indecomposable continuum $Y$ and a light map $f: Y \longrightarrow X$. Let $W: \mathcal{C}(Y) \longrightarrow \mathbb{R}^{+}$be a Whitney map for $\mathcal{C}(Y)$. Let $0<t \leq W(Y)$. $W^{-1}(t)$ is a closed subset of $\mathcal{C}(Y)$. Since $Y$ is hereditarily indecomposable $W^{-1}(t)$ is a decomposition of $Y$ (see 1.78, p. 123 of $[6])$. As $t>0, W^{-1}(t)$ does not contain singletons.

We make a remark before continuing with the proof of Theorem 2.1.

Remark. It can be shown that for sufficiently small $t>0, \operatorname{dim} W^{-1}(t)=\infty$. (This is obtained in the proof of the fact that $\operatorname{dim} \mathcal{C}(Y)=\infty$ for 2-dimensional hereditarily indecomposable continuum $Y$. See [6].) And one is tempted to apply the map $f^{*}: \mathcal{C}(Y) \longrightarrow \mathcal{C}(X)$ defined by $K \in \mathcal{C}(Y) \longrightarrow f(K) \in \mathcal{C}(X)$ to show that $\operatorname{dim} f^{*}\left(W^{-1}(t)\right)=\infty$. But it seems as if $f^{*}$ may fail to be light or even finite dimensional on $W^{-1}(t)$, and we need Lemma 1.3 to proceed. 
Return to the proof of Theorem 2.1. We may apply Lemma 1.3 to $\mathcal{K}=W^{-1}(t)$ since $W^{-1}(t)$ is closed in $\mathcal{C}(Y)$. If $\mathcal{C}(X)$ is finite dimensional then by Lemma 1.3 for every $\epsilon>0$ there exists a closed subset $Z$ of $Y$ with $d_{1}(Z)<\epsilon$ such that $Z$ intersects each member of $W^{-1}(t)$. As $\operatorname{dim} Y=2$, by the observation in the end of Section 1 there exist closed disjoint subsets $F_{1}$ and $F_{2}$ of $Y$ and $r>0$ such that every closed subset $L$ of $Y$ which separates $F_{1}$ from $F_{2}$ in $Y$ must satisfy $d_{1}(L) \geq r$. Since $W^{-1}(0)$ is the space of singletons, it follows from compactness that there is a $t>0$ such that for each $A \in W^{-1}(t), \operatorname{diam} A<r$.

Set $\epsilon=\operatorname{dist} .\left(F_{1}, F_{2}\right)$, and let $Z \subset Y$ with $d_{1}(Z)<\epsilon$ be as above. As $d_{1}(Z)<\epsilon$ we can represent $Z$ as $Z=Z_{1} \cup Z_{2} \cup \ldots \cup Z_{m}$ with $Z_{i}$ mutually disjoint closed sets and $\operatorname{diam} Z_{i}<\epsilon$. Set $F_{1}^{\prime}=F_{1} \cup\left(\bigcup\left\{Z_{i}: Z_{i} \cap F_{1} \neq \emptyset\right\}\right)$ and $F_{2}^{\prime}=F_{2} \cup\left(\bigcup\left\{Z_{i}\right.\right.$ : $\left.\left.Z_{i} \cap F_{1}=\emptyset\right\}\right)$. $F_{1}^{\prime}$ and $F_{2}^{\prime}$ are disjoint and closed. Let $L$ be a closed subset of $Y$ which separates $F_{1}^{\prime}$ from $F_{2}^{\prime}$. Then $L$ also separates $F_{1}$ from $F_{2}$ and hence $d_{1}(L) \geq r$. Let $L_{1}$ be a component of $L$ with $\operatorname{diam} L_{1} \geq r$. As $L \cap Z=\emptyset, L_{1} \cap Z=\emptyset$ too. $W^{-1}(t)$ is a decomposition of $Y$; hence there exists some $A \in W^{-1}(t)$ such that $L_{1} \cap A \neq \emptyset$. As $\operatorname{diam} A<r$ and $Y$ is hereditarily indecomposable $A \subset L_{1}$. But $Z$ intersects each member of $W^{-1}(t)$, so $Z \cap A \neq \emptyset$ which contradicts $L \cap Z=\emptyset$.

Theorem 2.1 shows that $\operatorname{dim} \mathcal{C}(X)=\infty$. Actually more is true; a similar but slightly more subtle argument implies the following result.

Theorem 2.2. Let $X$ be a 2-dimensional continuum and let $W: \mathcal{C}(X) \longrightarrow \mathbb{R}^{+}$be a Whitney map. Then for all sufficiently small $t>0, \operatorname{dim} W^{-1}(t)=\infty$.

Proof. Let $Y$ be a 2-dimensional hereditarily indecomposable continuum and let $f: Y \longrightarrow X$ be light as in the proof of Theorem 1.1. Let $W: \mathcal{C}(X) \longrightarrow \mathbb{R}^{+}$be a Whitney map and let $0<t<W(f(Y))$. $W^{-1}(t)$ is a closed subset of $\mathcal{C}(X)$. Note that no two elements of $W^{-1}(t)$ contain one another. Let $\mathcal{K}_{0}=\{A: A \in$ $\left.\mathcal{C}(Y), f(A) \in W^{-1}(t)\right\}$. Each linearly ordered (by inclusion) subset of $\mathcal{K}_{0}$ has an upper bound in $\mathcal{K}_{0}$ (namely the closed union of the elements of the subset). Let $\mathcal{K}$ denote the set of all maximal members of $\mathcal{K}_{0}$.

$\mathcal{K}$ is a decomposition of $Y$. Indeed, if $A, B \in \mathcal{K}$ and $A \cap B \neq \emptyset$ then $A \subset B$ or $B \subset A$ ( as $Y$ is hereditarily indecomposable) and by the maximality of members of $\mathcal{K}$ we have $A=B$. Let $y \in Y . \mathcal{L}=\{A: A \in \mathcal{C}(Y), y \in A \subset Y\}$ is an arc in $\mathcal{C}(Y)$ with end points $\{y\}$ and $Y$. Then $\{f(A): A \in \mathcal{L}\}$ is an $\operatorname{arc} \operatorname{in} \mathcal{C}(X)$ joining $\{x\}=f(\{y\})$ and $f(Y)$. Hence, as $0<t<W(f(Y))$, there is some $A \in \mathcal{L}$ with $W(f(A))=t$. It follows that there is some $B \in \mathcal{K}$ with $y \in A \subset B$ which shows that $\mathcal{K}$ is a decomposition of $Y$.

Note also that as $f$ is light and $W^{-1}(t)$ does not contain $\operatorname{singletons}, \inf \{\operatorname{diam} K$ : $K \in \mathcal{K}\}>0$ and that for every $r>0$ there is $t_{r}>0$ such that $0<t \leq t_{r}$ implies that the diameter of each element of $\mathcal{K}$ is less than $r$.

To apply Lemma 1.3 we have to check that $g$ is continuous. Indeed let a sequence $y_{1}, y_{2}, \ldots \in Y$ converge to $y_{0} \in Y$ and let $y_{i} \in K_{i} \in \mathcal{K}$. Since $\mathcal{C}(Y)$ is compact one can find a subsequence $K_{i_{j}}, i_{j}>0$, converging to $K \in \mathcal{C}(Y)$ in $2^{Y}$. Then $y_{0} \in K$ and as $Y$ is hereditarily indecomposable $K \subset K_{0}$ or $K_{0} \subset K$. It is clear that $f\left(K_{i_{j}}\right) \longrightarrow f(K)$ in $2^{X}$ and since $W^{-1}(t)$ is closed, $f(K) \in W^{-1}(t)$. So by the maximality of $K_{0}, K \subset K_{0}$ and since no two elements of $W^{-1}(t)$ contain one another $f\left(K_{0}\right)=f(K)$. Thus $g\left(y_{i_{j}}\right)=f\left(K_{i_{j}}\right) \longrightarrow f(K)=f\left(K_{0}\right)=g\left(y_{0}\right)$ which implies that $g$ is continuous. 
Clearly $g(Y) \subset W^{-1}(t)$. So assuming that $W^{-1}(t)$ is finite dimensional for some sufficiently small $t>0$ we can apply Lemma 1.3 and obtain a contradiction as in the proof of Theorem 2.1.

Remark. Actually the decomposition $\mathcal{K}$ constructed in Theorem 2.2 and the decomposition $\mathcal{K}=W^{-1}(t)$ constructed in Theorem 2.1 are upper semicontinuous. Let $q: Y \longrightarrow \mathcal{K}$ be the corresponding quotient map and let $f^{*}: \mathcal{K} \longrightarrow \mathcal{C}(X)$ be defined by $K \longrightarrow f(K)$. (Clearly $g=f^{*} q$.) In Theorem $2.1 q$ is open but it seems that $f^{*}$ may fail to be light or even finite dimensional. It can be shown that in Theorem 2.2 $f^{*}$ is light but it seems that $q$ may fail to be open. So assuming that $\mathcal{C}(X)$ is finite dimensional we are not able in the both cases to apply Kelley's theorem (Theorem 7.8 of [4]) to $q$ and we need Lemma 1.3.

\section{REFERENCES}

1. R. H. Bing, Higher-dimensional hereditarily indecomposable continua, Trans. AMS, 71(1951), 267-273. MR 13:265c

2. A. N. Dranishnikov, D. V. Repovs and T. V. Schepin, On approximation and embedding problems for cohomological dimension, Top. and Appl., 55(1994), 67-86. MR 94m:55001

3. H. Kato, The dimension of hyperspaces of certain 2-dimensional continua, Top. and Appl., 28(1988), 83-87. MR 89b:54012

4. J. L. Kelley, Hyperspaces of a continuum, Trans. AMS, 52(1942), 22-36. MR 3:315b

5. K. Kuratowski, Topology, Vol. 2, Academic Press, 1968. MR 41:4467

6. S. B. Nadler Jr., Hyperspaces of sets, Marcel Dekker, 1978. MR 58:18330

Department of Mathematics, Haifa University, Mount Carmel, Haifa 31905, Israel

E-mail address: levin@mathcs2.haifa.ac.il

E-mail address: yaki@mathcs2.haifa.ac.il 